\title{
Role of Escherichia coli YbeY, a highly conserved protein, in rRNA processing
}

Bryan W. Davies, ${ }^{1}$ Caroline Köhrer, ${ }^{1}$ Asha I. Jacob, ${ }^{1 \dagger}$ Lyle A. Simmons, ${ }^{1,2 \dagger}$ Jianyu Zhu, ${ }^{3}$

Lourdes M. Aleman, ${ }^{1}$ Uttam L. RajBhandary ${ }^{1}$ and Graham C. Walker ${ }^{1 *}$

${ }^{1}$ Department of Biology, Massachusetts Institute of Technology, Cambridge, MA 02139, USA.

${ }^{2}$ Department of Molecular, Cellular, and Development Biology, University of Michigan, Ann Arbor, MI, USA.

${ }^{3}$ Department of Molecular, Cell and Developmental Biology and Center for Molecular Biology of RNA, University of California at Santa Cruz, Santa Cruz, CA, USA.

\section{Summary}

The UPF0054 protein family is highly conserved with homologues present in nearly every sequenced bacterium. In some bacteria, the respective gene is essential, while in others its loss results in a highly pleiotropic phenotype. Despite detailed structural studies, a cellular role for this protein family has remained unknown. We report here that deletion of the Escherichia coli homologue, YbeY, causes striking defects that affect ribosome activity, translational fidelity and ribosome assembly. Mapping of 16S, $23 \mathrm{~S}$ and $5 \mathrm{~S}$ rRNA termini reveals that $\mathrm{YbeY}$ influences the maturation of all three rRNAs, with a particularly strong effect on maturation at both the $5^{\prime}$ - and $3^{\prime}$-ends of $16 \mathrm{~S}$ rRNA as well as maturation of the $5^{\prime}$-termini of 23S and 5S rRNAs. Furthermore, we demonstrate strong genetic interactions between ybeY and rnc (encoding RNase III), ybeY and $r n r$ (encoding RNase $\mathrm{R}$ ), and ybeY and pnp (encoding PNPase), further suggesting a role for YbeY in rRNA maturation. Mutation of highly conserved amino acids in $\mathrm{YbeY}$, allowed the identification of two residues (H114, R59) that were found to have a significant effect in vivo. We discuss the implications of these findings for rRNA maturation and ribosome assembly in bacteria.

Accepted 13 August, 2010. *For correspondence. E-mail gwalker@ mit.edu; Tel. (+ 617) 253 6711; Fax (+617) 253 2643. ${ }^{+}$These authors contributed equally to this work.

\section{Introduction}

Ribosome maturation and assembly occur in a cooperative and ordered fashion (Noller and Nomura, 1987; Kaczanowska and Ryden-Aulin, 2007). In bacteria, a $50 S$ ribosomal subunit associates with a $30 \mathrm{~S}$ ribosomal subunit to form an active $70 S$ ribosome. The $50 S$ ribosomal subunit is comprised of $23 S$ and $5 S$ rRNAs and 33 ribosomal proteins, while the $30 S$ ribosomal subunit is comprised of $16 \mathrm{~S}$ rRNA and 21 ribosomal proteins (Wilson and Nierhaus, 2007). 16S, 235 and 5S rRNAs are cotranscribed as part of a large rRNA precursor. Before transcription is complete, ribosomal proteins associate with rRNA to form ribonucleoprotein complexes that are acted on by RNase III (Robertson et al., 1967). RNase III cleaves the initial transcript into 17S, $25 \mathrm{~S}$ and $9 \mathrm{~S}$ rRNA precursors that undergo further processing at their $5^{\prime}$ - and $3^{\prime}$-termini to yield mature $16 S$, $23 \mathrm{~S}$ and 5S rRNAs respectively (Gegenheimer et al., 1977; Nierhaus, 1991).

Our understanding of the steps by which rRNA precursors are processed to their mature forms is still incomplete. In Escherichia coli, RNase G and RNase E are required for the maturation of the $5^{\prime}$-terminus of $16 \mathrm{~S}$ rRNA (Li et al., 1999a). RNase T is required for maturation of the 3'-terminus of $23 \mathrm{~S}$ rRNA (Li et al., 1999b). RNase E is required for partial maturation of the $5^{\prime}$-terminus of $5 S$ rRNA (Misra and Apirion, 1979), and RNase E and RNase $T$ are required for the maturation of the $3^{\prime}$-terminus of $5 S$ rRNA (Misra and Apirion, 1979; Li and Deutscher, 1995). RNases responsible for the final step(s) in maturation of the $3^{\prime}$-terminus of $16 \mathrm{~S}$ rRNA, $5^{\prime}$-terminus of $23 \mathrm{~S}$ rRNA and $5^{\prime}$-terminus of $5 S$ rRNA remain unknown (Deutscher, 2009) (Fig. 1).

Reconstitution of active $30 \mathrm{~S}$ and $50 \mathrm{~S}$ ribosomal subunits has been performed in vitro using mature rRNAs and ribosomal proteins (Nomura and Erdmann, 1970; Wireman and Sypherd, 1974; Nierhaus, 1980; Green and Noller, 1999). However, the conditions required for in vitro reconstitution are far from physiological and it is well recognized that many additional proteins, which include processing, modification and assembly factors, are required for rRNA maturation and eventual 50S, $30 \mathrm{~S}$ and $70 \mathrm{~S}$ ribosome formation in vivo. Several of these factors, such as Era and ObgE, are essential for cell viability (Wilson 


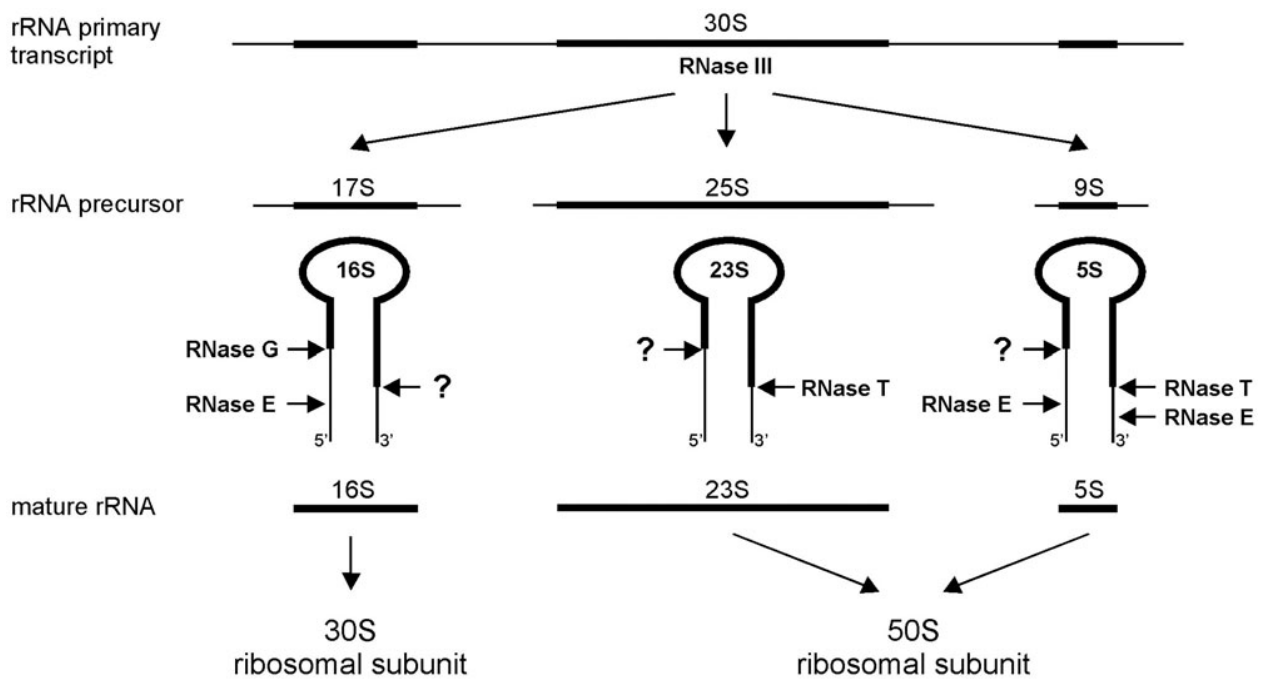

Fig. 1. Steps in the processing of rRNA in E. coli. All enzymes indicated are endonucleases with the exception of RNase T that generates mature $3^{\prime}$-termini by trimming the rRNA precursors.

and Nierhaus, 2007). While these accessory factors are clearly important, in many cases we lack a good understanding of their role in ribosome biogenesis (Bunner et al., 2010).

The UPF0054 protein family is highly conserved among prokaryotes. A gene encoding a member of this protein family is found in almost all sequenced bacterial genomes and it is one of the 206 genes that comprise the predicted minimal bacterial genome set (Gil et al., 2004). In some bacteria including Bacillus subtilis (Kobayashi et al., 2003), Haemophilus influenzae (Akerley et al., 2002) and Vibrio cholerae (B.W. Davies et al., in preparation), the respective homologue is essential. In the plant symbiont, Sinorhizobium meliloti, the respective homologue is not essential, but loss of its activity results in extreme pleiotropy and an inability of $S$. meliloti to form a symbiosis with plant hosts (Davies and Walker, 2008).

Here, we show that loss of the $E$. coli homologue, YbeY, results in viable cells with a pleiotropic phenotype and striking defects in ribosome function including decreased ribosome activity, reduced translational fidelity and altered translation initiation factor binding. Furthermore, we demonstrate that deletion of ybeY strongly affects the maturation of all three rRNAs causing a particularly strong defect in maturation of the 16S rRNA $5^{\prime}$ and $3^{\prime}$-termini, as well as maturation of the $5^{\prime}$-termini of 23S and $5 S$ rRNAs. Strong genetic interactions between $y b e Y$ and $r n c$ (encoding RNase III), ybeY and $r n r$ (encoding RNase R), and ybeY and pnp (encoding PNPase) additionally suggest a requirement for YbeY activity in rRNA maturation. We discuss the implications of these findings for rRNA maturation and ribosome assembly in bacteria.

Results

\section{UPF0054 protein family members in bacteria are functionally equivalent}

Disruption of the gene encoding the $S$. meliloti UPF0054 protein family member, $S M C 01113$, results in extreme pleiotropy (Davies and Walker, 2008). Deletion of the $E$. coli UPF0054 family member gene, $y b e Y$, from the reference strain MC4100 similarly results in an extremely pleiotropic phenotype that includes a modest decrease in growth rate in rich medium (Fig. $2 \mathrm{~A}$ ), but a significant sensitivity to numerous physiologically diverse stresses including $\beta$-lactam antibiotics, temperature, detergents and oxidative stress (Fig. 2B and C; Fig. S1). Deletion of ybeY from a second reference E. coli strain, MG1655, resulted in the same phenotypes (data not shown), indicating that the effects are not strain-specific. All $\Delta y b e Y$ mutant phenotypes were rescued by ectopic expression of ybeY (Fig. 2A-C; Fig. S1). Expression of either the $S$. meliloti homologue SMc01113 or the B. subtilis homologue $y q f G$ rescues the $\triangle y b e Y$ mutant phenotypes as effectively as $y b e Y$ itself (Fig. 2A-C; Fig. S1). Conversely, expression of $y b e Y$ in the S. meliloti SMc01113 mutant background rescues all free-living and symbiotic phenotypes of the SMc01113 mutant (Fig. S2A-C and data not shown) (Jones et al., 2007; Davies and Walker, 2008). Taken together, these results demonstrate a universally conserved function for UPF0054 members in bacteria. 
A

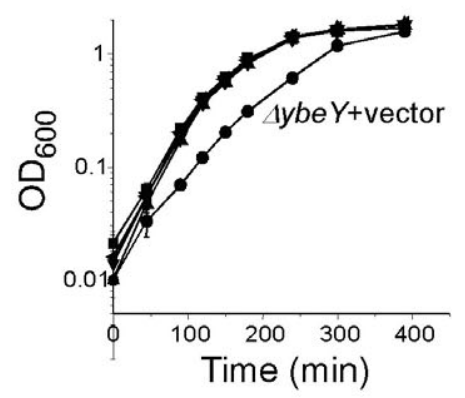

D

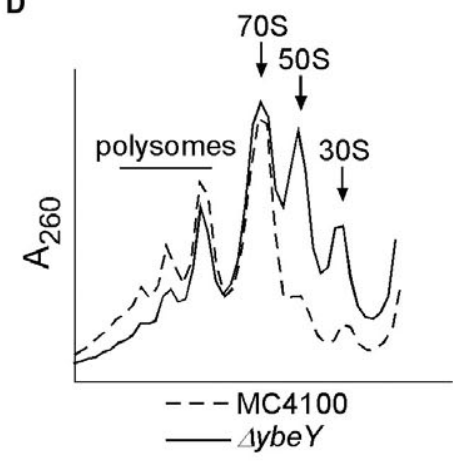

G

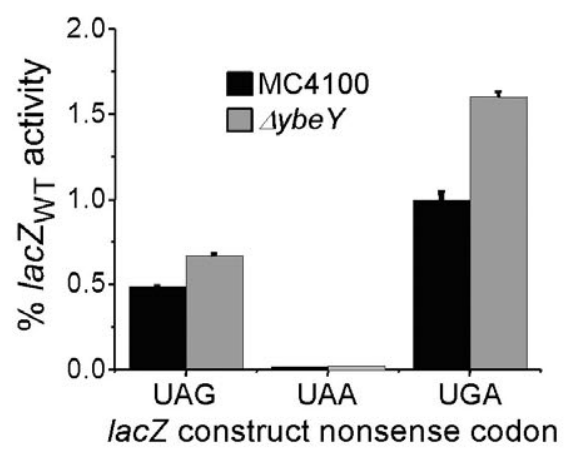

B

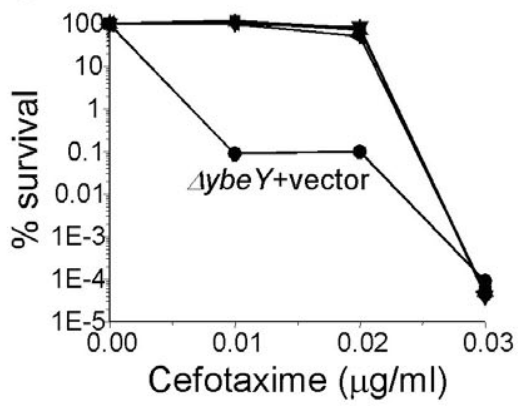

C

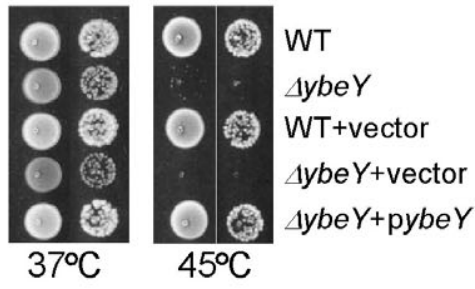

E

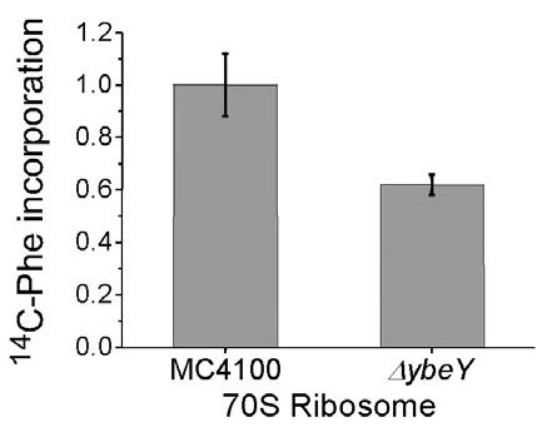

H
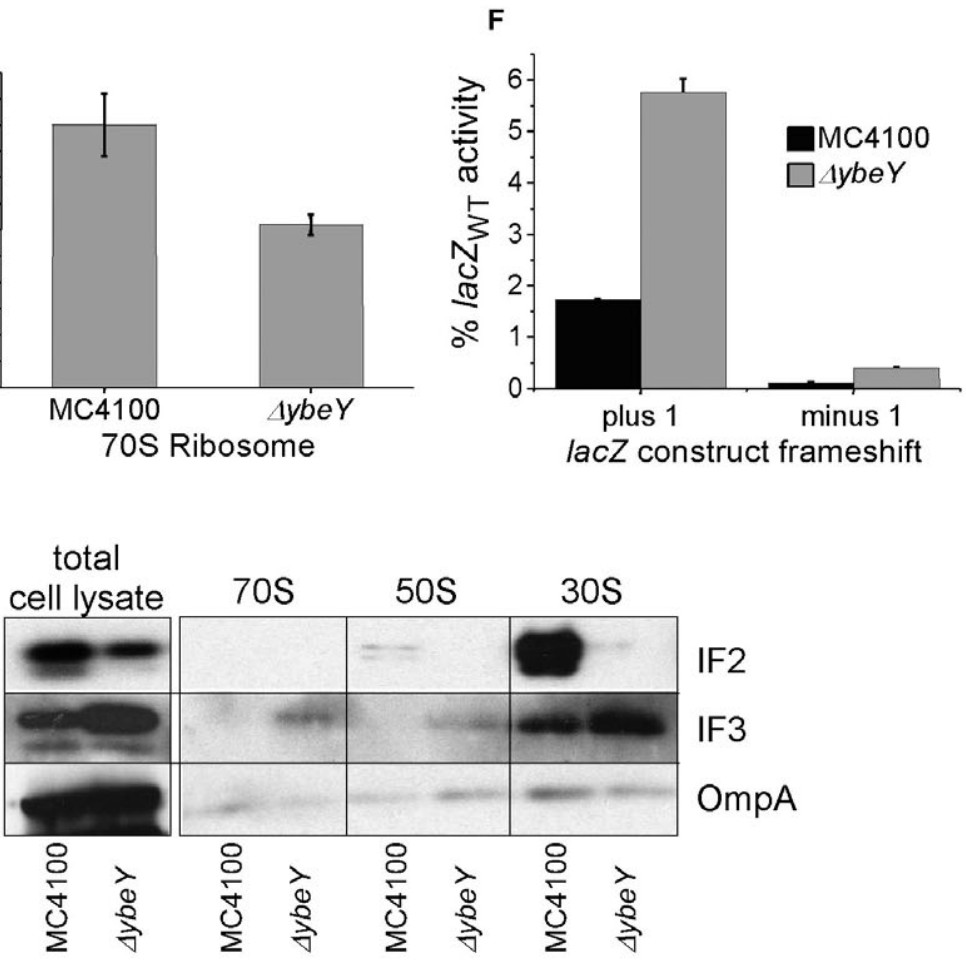

Fig. 2. Phenotypic analysis of the E. coli $\Delta y b e Y$ mutant.

A-C. Growth curves of MC4100 (WT) and the $\Delta y b e Y$ mutant complemented strains in LB at $37^{\circ} \mathrm{C}$. Doubling times: $40 \pm 2$ min ( $\Delta y b e Y$ mutant) vs. $28 \pm 3 \mathrm{~min}(\mathrm{MC} 4100)$. Sensitivity of the $\Delta y b e Y$ mutant to stresses (B) cefotaxime and (C) temperature. The $\Delta y b e Y$ mutant with empty vector only $(\Delta y b e Y+$ vector $)$ is shown on each plot for clarity. UPF0054 homologues: ybeY (E. coli), yqfG (B. subtilis) and SMc01113 (S. meliloti). WT + vector $(\square), \Delta y b e Y+$ vector $(\bullet), \Delta y b e Y+\operatorname{pybe} Y(\mathbf{\Delta}), \Delta y b e Y+$ pyqfG $(\boldsymbol{\nabla})$ and $\Delta y b e Y+p S M c 01113(\varangle)$. ' $p$ ' indicates that the gene indicated is expressed from a plasmid. In (C), the pairs of samples show a 10-fold dilution each.

D. Polysome profiles for MC4100 and the $\Delta y b e Y$ mutant. The positions of polysomes, $70 \mathrm{~S}$ ribosomes and $50 \mathrm{~S}$ and $30 \mathrm{~S}$ ribosomal subunits are indicated.

E-G. In vitro translation assay under saturating substrate conditions. MC4100 S100 fractions were mixed with a polyU template and equal amounts of MC4100 or $\Delta y b e Y$ mutant $70 S$ ribosomes in an in vitro translation reaction as described in the Experimental procedures. Translational activity is normalized to MC4100 70 S ribosome reactions. MC4100 and the $\Delta y b e Y$ mutant were transformed with plasmids expressing lacZ containing ( $F)$ frameshift mutations $(+1$ or -1$)$ or $(G)$ nonsense codons. LacZ activity was assayed as described in the Experimental procedures. The per cent LacZ activity (in Miller units) of each mutant lac $Z$ allele relative to the wild-type lac $Z$ allele for MC4100 or the $\Delta y b e Y$ mutant is reported. The value of LacZ activity from the wild-type allele (18921 Miller units in the $\Delta y b e Y$ mutant and 12247 Miller units in MC4100, respectively) was set to as $100 \%$ activity. For clarity, wild-type LacZ activity has been omitted from the plots. Each assay was performed in triplicate.

H. Immunoblots identifying IF2 and IF3 in whole cell lysates, 30S, 50S and 70S ribosome fractions from MC4100 and the $4 y b e Y$ mutant. Immunoblotting for OmpA is used as a loading control. Equal $\mathrm{A}_{260}$ amounts were loaded for the $30 \mathrm{~S}, 50 \mathrm{~S}$ and $70 \mathrm{~S}$ ribosome fractions. The experiment was repeated three times and a representative result is shown. 
Deletion of ybeY causes defects in polysome formation, ribosome activity, translational fidelity and translation initiation factor binding

Compared with the polysome profile of the parental strain, the $\Delta y b e Y$ mutant has a decrease in polysomes and a large increase in both free $50 \mathrm{~S}$ and free $30 \mathrm{~S}$ ribosomal subunits relative to $70 \mathrm{~S}$ ribosomes (Fig. 2D). Ectopic expression of $y b e Y$ in the $\triangle y b e Y$ mutant restored the normal polysome profile (Fig. S3A-C). After we had completed much of this work, a study investigating the role of $\mathrm{YbeY}$ in the $E$. coli heat shock response also reported that deletion of $y b e Y$ caused loss of polysomes (Rasouly et al., 2009); however, there was no mention of a potential function for YbeY in ribosome maturation.

The decreased polysome population led us to examine the functional state of the intact ribosomes in the $\Delta y b e Y$ mutant. We reconstituted an in vitro translation system with an $\mathrm{S} 100$ extract from the wild-type strain MC4100 and 70S ribosomes from either the $\Delta y b e Y$ mutant or its parent MC4100. Under saturating substrate conditions, ribosomes from the $\Delta y b e Y$ mutant had reduced translational activity compared with $70 \mathrm{~S}$ ribosomes from MC4100 (Fig. 2E). Furthermore, both frameshifting and readthrough of nonsense codons were increased in vivo in the $\triangle y b e Y$ mutant, with the largest effect being for +1 frameshifts (Fig. $2 F$ and G). The accumulation of defects in ribosomal function and polysome assembly, could account for the pleiotropic nature of the $\Delta y b e Y$ mutant as many stress response programmes rely on the upregulation of defence proteins to ward off stress or to repair damage (Imlay and Linn, 1987; Friedberg et al., 2005).

The increased pools of free $30 \mathrm{~S}$ and $50 \mathrm{~S}$ ribosomal subunits found in the $\triangle y b e Y$ mutant suggest that a portion of these ribosomes may be defective in assembly of the $70 \mathrm{~S}$ ribosome. In bacteria, translation initiation requires three protein initiation factors; IF1, IF2 and IF3. IF2 correctly positions the initiator tRNA in the ribosomal $\mathrm{P}$ site and promotes association of $30 S$ and $50 S$ ribosomal subunits. While IF1 is also known to stabilize the binding of the initiator tRNA, IF3 acts as a proofreading and anti-association factor (Kaczanowska and Ryden-Aulin, 2007). Analysis of total cell lysates showed only a slight but reproducible decrease in IF2 and an increase in IF3 in the $\Delta y b e Y$ mutant (Fig. 2H). However, we found a substantial decrease in IF2 present in the fraction containing $30 \mathrm{~S}$ subunits isolated from the $\Delta y b e Y$ mutant, suggesting that many of the mutant $30 \mathrm{~S}$ ribosomal subunits are not available for $70 S$ ribosome assembly. We also observed a slight increase in IF3 associated with $\triangle y b e Y$ mutant $30 \mathrm{~S}$ subunits and $70 S$ ribosomes, but the change was modest compared with IF2. This suggests that the $30 \mathrm{~S}$ ribosomal subunits in a $\Delta y b e Y$ mutant have a defect that prevents ribosome assembly and/or promotes disassembly of $70 \mathrm{~S}$ ribosomes when assembly is attempted.

\section{The $\triangle \mathrm{ybeY}$ mutant is defective in rRNA maturation}

The ribosome is primarily composed of rRNA (Wilson and Nierhaus, 2007) and incomplete processing of rRNA can lead to defects in ribosome function (Wireman and Sypherd, 1974). Comparison of total rRNA profiles by agarose gel electrophoresis from MC4100 and the $\Delta y b e Y$ mutant showed the presence of a substantial amount of $17 \mathrm{~S}$ rRNA in the $\triangle y b e Y$ mutant, as well as a faster migrating species, which we annotate as $16 \mathrm{~S}^{*}$, indicating a defect in 16S rRNA maturation (Fig. 3A). 17S rRNA is the precursor of 16S rRNA (Fig. 1) and is generated from the initial rRNA transcript by RNase III cleavage (Gegenheimer et al., 1977). The aberrant $16 S^{*}$ rRNA species may be a product of misprocessing and/or degradation of the $17 S$ or 16S rRNA. Ectopic expression of $y b e Y$ in the $\triangle y b e Y$ mutant restored normal $16 S$ rRNA maturation (Fig. S3D). We were unable to assess the state of $5 S$ and $23 S$ rRNA processing in these total rRNA profile experiments (see below, however) because the difference between mature and immature $5 S$ and $23 S$ rRNAs is only a few nucleotides, respectively, and thus does not cause a detectable shift on agarose gels.

In strain MC4100, 30S ribosomal subunits contained mostly $16 \mathrm{~S}$ rRNA along with some $17 \mathrm{~S}$ rRNA, whereas 70 S ribosomes contained only 16S rRNA (Fig. 3A). In contrast, $30 \mathrm{~S}$ ribosomal subunits from the $\triangle y b e Y$ mutant contained much more 17S rRNA than 16S rRNA as well as a substantial amount of $16 S^{*}$ rRNA (Fig. 3A). Furthermore, there was also a substantial amount of 17S rRNA in $70 \mathrm{~S}$ ribosomes isolated from the $\triangle y b e Y$ mutant (Fig. 3A). The accumulation of 17S rRNA was not due to the slower growth rate of the $\triangle y b e Y$ mutant. When the strains were grown in minimal medium where MC4100 and the $\triangle y b e Y$ mutant show similar doubling times, we still observed similar levels of $17 \mathrm{~S}$ rRNA accumulation (data not shown). 17S rRNA is thought not to be competent for translation in vitro (Wireman and Sypherd, 1974). The increased abundance of $17 \mathrm{~S}$ and $16 \mathrm{~S}^{*}$ rRNA in the $30 \mathrm{~S}$ ribosomal subunits could account for altered translation initiation factor binding and the other translational defects described above for the $\triangle y b e Y$ mutant. Similarly, the increased abundance of $17 \mathrm{~S}$ rRNA in $70 \mathrm{~S}$ ribosomes could explain, at least in part, the reduced translation activity and decreased translational accuracy of the $\Delta y b e Y 70 S$ ribosomes. Interestingly, $16 \mathrm{~S}^{*}$ rRNA was not found in $\triangle y b e Y 70 S$ ribosomes suggesting that it is not competent for assembly into the $70 \mathrm{~S}$ ribosome and may thus be a non-functional product of rRNA processing in the $\Delta y b e Y$ mutant. 
A

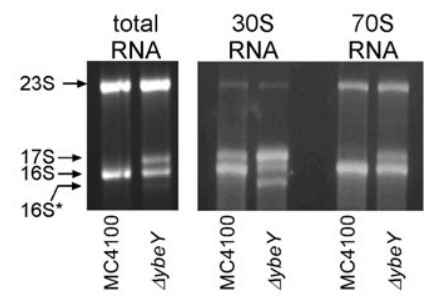

C

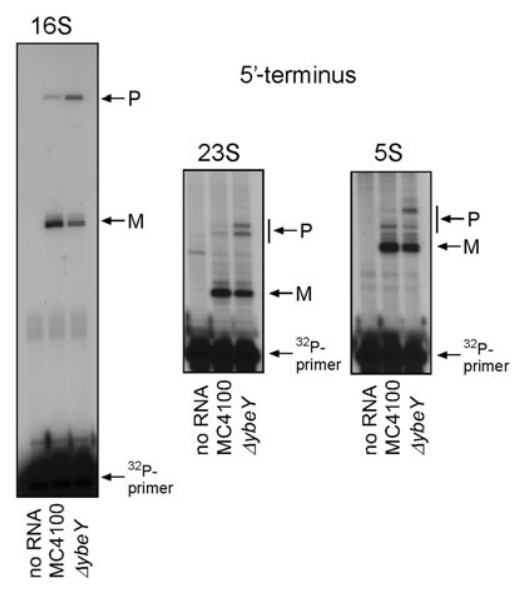

B

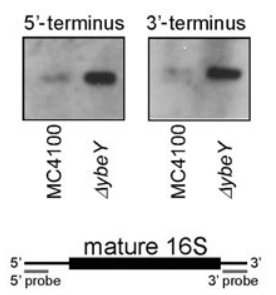

17S precursor 3'-terminus

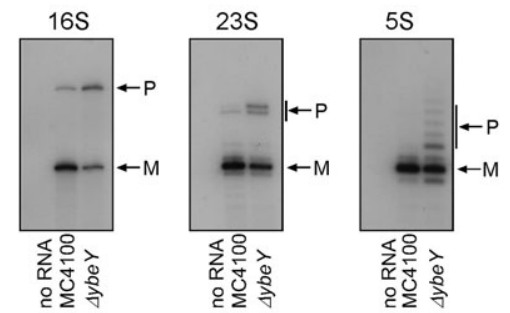

Fig. 3. Analysis of rRNA from E. coli MC4100 and the $\triangle y b e Y$ mutant.

A. Total RNA isolated from whole cells, 30S ribosomal subunits and $70 \mathrm{~S}$ ribosomes from MC4100 and the $\Delta y b e Y$ mutant. The positions of $23 \mathrm{~S}, 17 \mathrm{~S}, 16 \mathrm{~S}$ and $16 \mathrm{~S}^{\star}$ rRNAs are indicated based on their mobility.

B. Northern blot analysis using probes directed against the $5^{\prime}$ - and $3^{\prime}$-termini of $17 S$ rRNA. Equal amounts of total RNA from MC4100 and the $\Delta y b e Y$ mutant were used. The locations of the probes are shown in the diagram below the blots.

$\mathrm{C}$ and D. Primer extension and site-specific RNase $\mathrm{H}$ cleavage assays to map the $5^{\prime}$ - and 3 '-termini of 16S, 23S and 5S rRNAs from MC4100 and the $\triangle y b e Y$ mutant. ' $P$ ' and ' $M$ ' indicate the location of bands corresponding to precursor and mature forms of the rRNA respectively. Annotation of the positions of the $5^{\prime}$ and $3^{\prime}$-termini of $16 \mathrm{~S}, 23 \mathrm{~S}$ and $5 \mathrm{~S}$ rRNAs, mature and precursor species, were based on previous observations (Li et al., 1999a). Total RNA was prepared from MC4100 and the $\Delta y b e Y$ mutant strains as described in the Experimental procedures.
In agreement with the observed increase in 17S rRNA in the $\Delta y b e Y$ mutant, Northern blot analysis showed that both $5^{\prime}$ - and $3^{\prime}$-termini of $17 \mathrm{~S}$ rRNA were present at much higher levels in total RNA extracted from the $\Delta y b e Y$ mutant than in total RNA from the parental strain MC4100 (Fig. 3B). Use of primer extension to map the $5^{\prime}$-terminus (Fig. 3C) and site-specific RNase $\mathrm{H}$ cleavage followed by Northern hybridization (Li et al., 1999b) to map the 3 '-terminus of 16S rRNA (Fig. 3D) showed that the termini of the mature and immature 16S rRNA in total RNA extracted from the $\Delta y b e Y$ mutant and the parental strain MC4100 were identical. However, consistent with the above results, both immature $5^{\prime}$ - and 3 '-termini of $16 S$ rRNA were present at higher levels in total RNA extracted from the $\triangle y b e Y$ mutant (Fig. $3 C$ and D). We did not observe either $5^{\prime}$ - or $3^{\prime}$-termini shorter than that of mature $16 \mathrm{~S}$ rRNA, although, if the $5^{\prime}$ - or $3^{\prime}$-terminus of the $16 \mathrm{~S}^{*}$ species were extensively degraded, the assays used would not have detected them.

Primer extension and site-specific RNase $\mathrm{H}$ cleavage were also used to determine the maturation state of $23 S$ and $5 S$ rRNAs. Strikingly, along with $16 S$ rRNA, the maturation of the $5^{\prime}$ - and $3^{\prime}$-termini of both $23 S$ and $5 S$ rRNA were also affected, with all four termini showing increased amounts of the immature form in the $\Delta y b e Y$ mutant (Fig. $3 C$ and $D$ ), indicating that $y b e Y$ function is required for normal $5^{\prime}$ and $3^{\prime}$ processing of $16 \mathrm{~S}, 23 \mathrm{~S}$ and $5 \mathrm{~S}$ rRNAs. Nevertheless, the most significant defects in the
$\Delta y b e Y$ mutant appeared to be in the complete maturation of the $5^{\prime}$ - and $3^{\prime}$-termini of $16 \mathrm{~S}$ rRNA.

Rasouly et al. demonstrated a defect in the polysome profile of a $\Delta y b e Y$ mutant after heat shock (Rasouly et al., 2009). After shifting the cultures to $45^{\circ} \mathrm{C}$ for $1 \mathrm{~h}$, we observed little mature $16 \mathrm{~S}$ rRNA in the $\triangle y b e Y$ mutant by agarose gel electrophoresis; instead we saw two bands that migrate near the $17 \mathrm{~S}$ and $16 \mathrm{~S}^{*}$ rRNA positions (Fig. S3E). In contrast, there were no significant changes in the rRNA profile of $\mathrm{MC} 4100$ samples shifted to $45^{\circ} \mathrm{C}$ compared with those at $37^{\circ} \mathrm{C}$. As $17 \mathrm{~S}$ rRNA is not competent for translation (Wireman and Sypherd, 1974), and 16S* rRNA appears to be excluded from 705 ribosomes (Fig. 3A), the increase in 17S and 16S* rRNA-like species and the decrease in 16S rRNA in the $\triangle y b e Y$ mutant following heat shock could explain the defect in polysome formation at elevated temperatures (Rasouly et al., 2009) and the overall temperature sensitivity of the $\Delta y b e Y$ mutant described above (Fig. 2C).

ybeY shows strong genetic interactions with rnc (RNase III), rnr (RNase R) and pnp (PNPase)

The serious defects in translation and rRNA maturation in the $\Delta y b e Y$ mutant suggested that $Y b e Y$ might be involved in rRNA metabolism. To explore the cellular role of YbeY, we compared the rRNA profile of the $\triangle y b e Y$ mutant with that of several well-characterized E. coli RNase mutants 
A

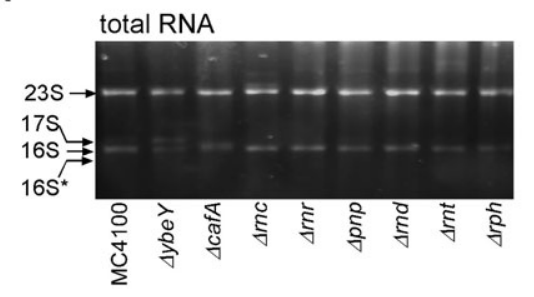

C

$16 \mathrm{~S}$ - 5'-terminus
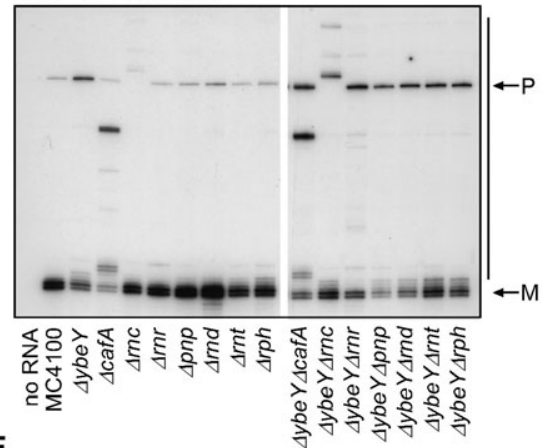

E $23 S$ - 5'-terminus
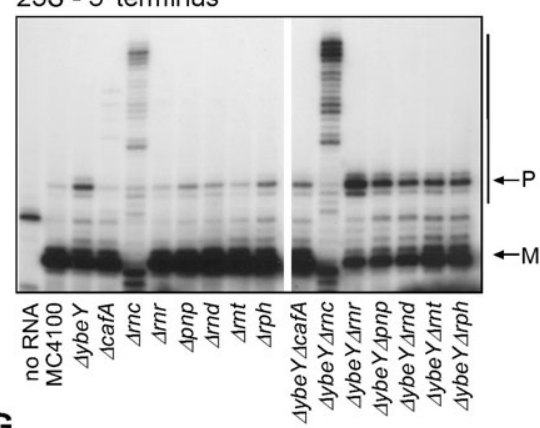

G

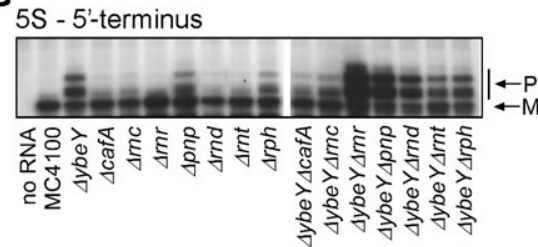

B

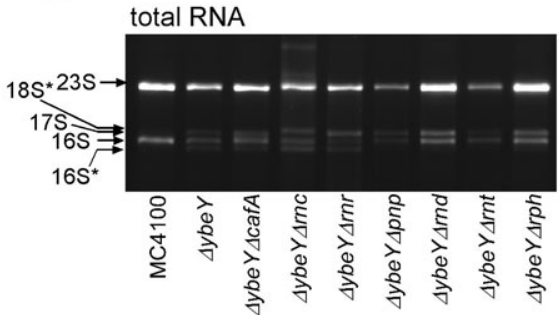

D

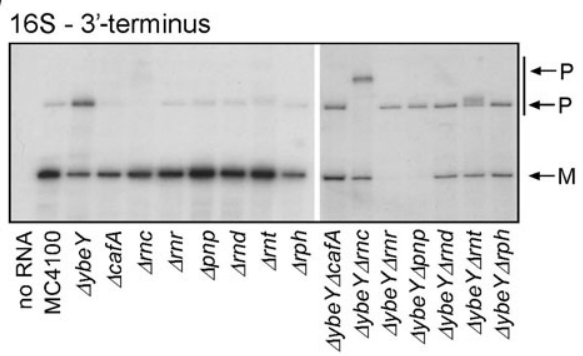

F

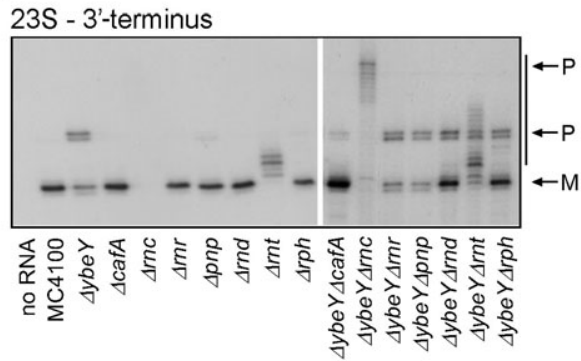

H

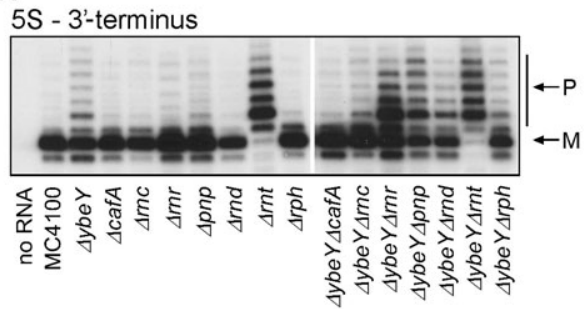

Fig. 4. Analysis of rRNA from the $\triangle y b e Y$ mutant and seven well-characterized $E$. coli RNase mutant strains. The relevant genotype from which the rRNA was extracted is indicated under each lane. The parental strain MC4100 rRNA is shown in each case as a control. A and B. Agarose gel electrophoresis of total rRNA from single and double RNase mutant strains. The positions of $23 \mathrm{~S}, 18 \mathrm{~S}^{\star}, 17 \mathrm{~S}, 16 \mathrm{~S}$ and $16 S^{*}$ rRNAs are indicated.

$\mathrm{C}-\mathrm{H}$. Primer extension and site-specific RNase $\mathrm{H}$ cleavage assays to map the $5^{\prime}$ - and $3^{\prime}$-termini of $16 \mathrm{~S}, 23 \mathrm{~S}$ and $5 \mathrm{~S}$ rRNAs from single and double RNase mutant strains. 'P' and ' $\mathrm{M}$ ' indicate the position of bands corresponding to the precursor and mature form for each rRNA. Annotation of the positions of the $5^{\prime}$ - and 3 '-termini of $16 \mathrm{~S}, 23 \mathrm{~S}$ and $5 \mathrm{~S}$ rRNAs, mature and precursor species, were based on previous observations (Li et al., 1999a).

disrupted in the following genes: cafA (RNase G), rnc (RNase III), rnr (RNase R), pnp (PNPase), rnd (RNase D), rnt (RNase T) and rph (RNase PH) (Fig. 4A). We also transduced each of these RNase mutations into the $\triangle y b e Y$ mutant background and examined the rRNA profiles of these double mutants (Fig. 4B). We then mapped the $5^{\prime}$ - and 3 '-termini of $16 \mathrm{~S}, 23 \mathrm{~S}$ and $5 \mathrm{~S}$ rRNAs for each strain using primer extension and site-specific RNase $\mathrm{H}$ cleavage assays (Fig. $4 \mathrm{C}-\mathrm{H}$ ) as described above.

Profiles of total rRNA showed that, of all the mutants tested, the $\triangle y b e Y$ mutant had the greatest defect in maturation of $17 \mathrm{~S}$ rRNA to $16 \mathrm{~S}$ rRNA (Fig. 4A). Primer extension and site-specific RNase $\mathrm{H}$ cleavage assays confirmed this result showing high levels of both immature 
$5^{\prime}$ and $3^{\prime} 16 \mathrm{~S}$ rRNA termini in the $\triangle y b e Y$ mutant compared with the other RNase mutants (Fig. 4C and D). As previously described (Li et al., 1999a), the $\triangle$ cafA mutant also showed severe defects in maturation of the 16S rRNA 5 '-terminus. However, in contrast to the $\Delta y b e Y$ mutant, which accumulates the full 17S rRNA 5' precursor (+ 115 nucleotides), the $\triangle$ cafA mutant accumulates the shorter +66 nucleotides $5^{\prime}$ precursor (Fig. 4C). Also, of all the mutants tested, only the $\Delta y b e Y$ mutant showed accumulation of high levels of immature 16S rRNA 3 '-terminus (Fig. 4D).

The introduction of either the rnc or rnr mutation into the $\Delta y b e Y$ mutant resulted in major alterations in the gross rRNA profile of the $\Delta y b e Y$ mutant (Fig. 4B). Strikingly, 17S rRNA was no longer present in the $\Delta y b e Y \Delta r n c$ mutant, instead $16 S^{*}$ rRNA and a new rRNA species migrating more slowly than 17S rRNA accumulated. Primer extension and site-specific RNase $\mathrm{H}$ cleavage assays confirmed this new precursor, which we designate $18 S^{*}$ rRNA, as a $16 S$ rRNA derivative with additional nucleotides on both the $5^{\prime}$ - and $3^{\prime}$-termini of $17 \mathrm{~S}$ rRNA (Fig. 4C and D). Gegenheimer et al. reported a transient $18 \mathrm{~S}$ rRNA species in a $\Delta r n c$ mutant (Gegenheimer et al., 1977 ) that is matured to $16 \mathrm{~S}$ rRNA and has a similar mobility as the new precursor species we observe. We have not mapped the termini of $18 S^{*}$ rRNA or the $18 \mathrm{~S}$ species found by Gegenheimer et al. to the nucleotide level, but based on their relative mobility, we suggest that the $18 S^{*}$ rRNA observed in the $\Delta y b e Y \Delta r n c$ mutant may be $18 \mathrm{~S}$ rRNA. If $18 \mathrm{~S}^{*}$ and $18 \mathrm{~S}$ rRNA are identical, our data suggest that the absence of $Y b e Y$ influences the processing of $18 \mathrm{~S}$ rRNA to $16 \mathrm{~S}$ rRNA in a $\Delta r n c$ mutant. Further characterization of the $18 S^{*}$ rRNA species in the $\Delta y b e Y \Delta r n c$ mutant could be of much interest.

The $\Delta y b e Y \Delta r n r$ mutant showed a substantial decrease in 16S rRNA and reciprocal increase in both $17 \mathrm{~S}$ and $16 \mathrm{~S}^{*}$ rRNA (Fig. 4B). This result was confirmed by mapping of the $5^{\prime}$ - and $3^{\prime}$-termini (Fig. 4C and D). Furthermore, primer extension of $16 \mathrm{~S}$ rRNA $5^{\prime}$-termini showed an increase in a set of new rRNA species of minor intensity that migrated between $17 S$ and $16 S$ rRNAs. These new species may represent partially mature or non-functional 16S-like rRNA species. RNase $R$ has been shown to function as a scavenging RNase that removes non-functional rRNA from the cell (Cheng and Deutscher, 2003). Loss of this function is consistent with the appearance of 16S rRNA intermediates observed by primer extension in the $\Delta y b e Y \Delta r n r$ mutant. It also suggests that, in the absence of $y b e Y$, the cell misprocesses large amounts of 17S rRNA generating abnormal species that RNase $R$ normally helps to degrade.

Strikingly, we observed very little of the mature 16S rRNA 3 '-terminus in either the $\Delta y b e Y \Delta r n r$ mutant or the $\Delta y b e Y$ spnp mutant rRNA (Fig. 4D). Deletion of either rnr or pnp alone does not significantly affect the maturation of the 16S rRNA 3 '-terminus (Fig. 4D). The RNase(s) required for maturation of the 16S rRNA 3 '-terminus has not been identified (Deutscher, 2009). Our results suggest that PNPase and RNase R may act in maturation of the 16S rRNA 3'-terminus and their role may be modulated by YbeY. Interestingly, while both the $\Delta y b e Y \Delta r n r$ mutant and the $\Delta y b e Y \Delta p n p$ mutant affect maturation of the 16S rRNA 3 '-terminus, only the $\triangle y b e Y$ $\Delta r n r$ mutant exhibits additional defects in 16S rRNA metabolism as shown by the appearance of extra aberrant rRNA species (Fig. 4B and C). This suggests that RNase $R$ and YbeY interact in aspects of rRNA metabolism beyond 16S rRNA 3'-terminus maturation, such as quality control of rRNA.

Mapping of the $5^{\prime}$ - and $3^{\prime}$-termini showed that deletion of $y b e Y$ also affected $5^{\prime}$ maturation of $23 S$ and $5 S$ rRNAs (Fig. 4E and H). The $\Delta y b e Y$ mutant had a greater effect in 23S and 5S rRNA maturation than nearly all of the RNase mutants tested. As expected, deletion of $r n c$ abolishes formation of mature 23S rRNA 5'- and 3'-termini (King et al., 1984). Also as expected, deletion of rnt greatly inhibits maturation of the $23 \mathrm{~S}$ and $5 \mathrm{~S}$ rRNA 3 '-termini ( $\mathrm{Li}$ et al., 1999b). Interestingly, we have also found that in the $\Delta y b e Y \Delta r n t$ mutant the 17S rRNA precursor was extended by one or two nucleotides at the $3^{\prime}$-terminus (Fig. 4D), suggesting the possible involvement of RNase $\mathrm{T}$ in trimming the $17 \mathrm{~S}$ precursor at the $3^{\prime}$-terminus prior to complete $3^{\prime}$ maturation of $16 \mathrm{~S}$ rRNA. Deletion of pnp and rph also caused accumulation of $5^{\prime}$-immature $5 S$ rRNA precursor implicating a role of these two RNases in this step of rRNA maturation (Fig. 4G).

RNase $E$ is known to function in the maturation of the 5 '-terminus of 16S rRNA (Li et al., 1999a), and the 5'- and 3 '-termini of 5S rRNA (Misra and Apirion, 1979; Li and Deutscher, 1995). RNase $E$ is essential in $E$. coli and temperature-sensitive mutants have been used to explore its potential roles in rRNA maturation (Li et al., 1999a). Using the rne-1 temperature-sensitive RNase E allele, we constructed an rne-1 $\Delta y b e Y::_{\text {cat }}{ }^{\mathrm{R}}$ double mutant and attempted to explore their genetic interaction in rRNA processing at the non-permissive temperature $\left(45^{\circ} \mathrm{C}\right)$. The rRNA profile is shown (Fig. S3F); mapping of the $16 \mathrm{~S}$ rRNA termini showed that RNase E contributes to the maturation of the $5^{\prime}$-terminus of $16 \mathrm{~S}$ rRNA as expected ( $\mathrm{Li}$ et al., 1999a), but does not contribute significantly to the maturation of the $3^{\prime}$-terminus under the conditions used (data not shown). Unfortunately, it is difficult to interpret the results from the rne-1 $\Delta y b e Y::$ cat $^{R}$ double mutant grown at the non-permissive temperature because of the strong temperature sensitivity of the $\Delta y b e Y$ mutant by itself (Rasouly et al., 2009; see also Fig. 2C and Fig. $\mathrm{S} 3 \mathrm{E})$. Because of this, effects of the rne-1 mutation at $45^{\circ} \mathrm{C}$ are overshadowed by effects of the $\Delta y b e Y$ deletion. 


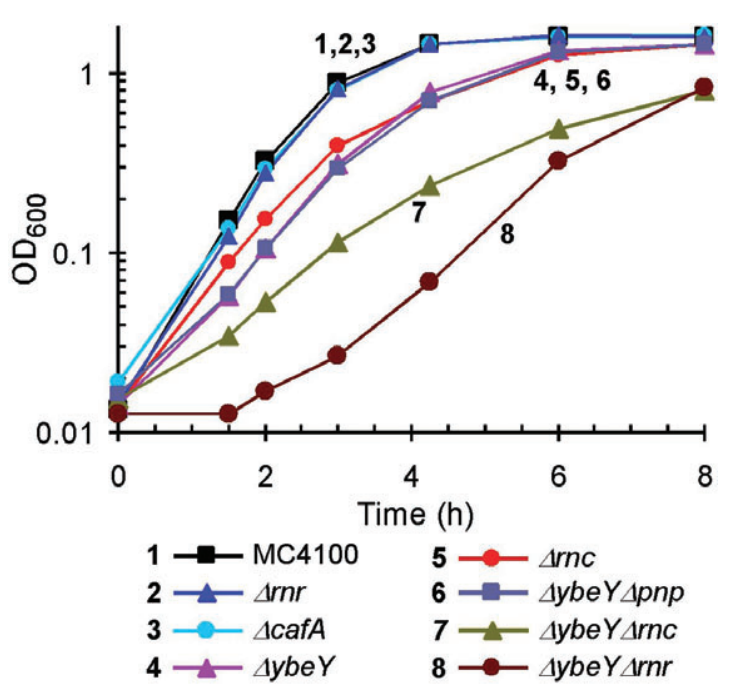

Fig. 5. Growth of MC4100, $\Delta y b e Y$ mutant and several $\Delta y b e Y$ double mutants in rich medium at $37^{\circ} \mathrm{C}$. Most $\Delta y b e Y$ double mutants did not show a growth defect (data not shown); the $\Delta y b e Y$ $\Delta p n p$ double mutant is shown as an example. In contrast, the $\Delta y b e Y \Delta r n c$ and $\Delta y b e Y \Delta r n r$ mutants showed a significant decrease in growth rate.

A temperature-independent approach for conditional rne inactivation will be necessary for such experiments.

\section{Growth rate analysis of the $\Delta y b e Y$ double mutants}

We next examined the growth behaviour in rich medium of single and double mutants described above. Further strengthening a connection between YbeY and RNase R, we observed the greatest effect in the $\Delta y b e Y \Delta r n r$ mutant (Fig. 5). This mutant shows reduced growth rate in addition to an extended lag phase. Interestingly, while both the $\Delta y b e Y \Delta r n r$ and the $\Delta y b e Y \Delta p n p$ mutants affect $16 \mathrm{~S}$ rRNA 3 -terminus maturation strongly, the $\Delta y b e Y \Delta p n p$ grows identically to the $\Delta y b e Y$ mutant, showing only a modest growth rate reduction. The change in growth behaviour of the $\Delta y b e Y \Delta r n r$ mutant compared with the $\Delta y b e Y$ mutant could be due to defects other than the incomplete maturation of the $16 \mathrm{~S}$ rRNA, e.g. quality control of rRNA. Also, the significant accumulation of $16 S^{*}$ rRNA, a species that can be assembled in $30 S$ ribosomal subunits, but not in functional 705 ribosomes (Fig. $3 \mathrm{~A}$ ) may be detrimental to the cell and be responsible for the growth behaviour of the $\Delta y b e Y \Delta r n r$ mutant.

We also observed a decrease in growth rate in the $\Delta y b e Y \Delta r n c$ mutant in rich medium (Fig. 5) compared with the respective single mutants, although this was less pronounced than for the $\Delta y b e Y \Delta r n r$ mutant. Thus, the strong genetic interaction observed in the $\Delta y b e Y \Delta r n r$ and $\Delta y b e Y$ $\Delta r n c$ mutants is manifested at both the rRNA processing and physiological levels.
Highly conserved amino acid residues required for $Y b e Y$ activity in vivo

All UPF0054 family members contain a conserved $\mathrm{H} 3 \mathrm{XH} 5 \mathrm{XH}$ motif (Fig. 6A). The presence of this motif has led to the categorization of UPF0054 family members as putative metal-dependent hydrolases and, more specifically, by some as putative metal-dependent proteases due to the presence of a similar motif found in certain mammalian proteases (E.V. Koonin, pers. comm.; Tatusov et al., 2001; Verma et al., 2002). The recently determined structures of $E$. coli YbeY and two additional UPF0054 family members (Oganesyan et al., 2003; Yeh et al., 2005; Zhan et al., 2005) support a putative metaldependent hydrolytic function; however, no substrate has been identified for this protein family despite extensive screening (Oganesyan et al., 2003).

To study the structure-function relationship of the conserved UPF0054 motif and other highly conserved amino acids in this protein family, we mutated several conserved amino acid residues in ybeY on our complementation plasmid to Ala (Fig. 6A). We then determined the ability of these mutant $y b e Y$ alleles to complement the growth and rRNA processing defects of the $\Delta y b e Y$ mutant (Fig. $6 \mathrm{~B}$ and $\mathrm{C})$. The first His residue $(\mathrm{H} 114)$ in the conserved $\mathrm{H} 3 \mathrm{XH} 5 \mathrm{XH}$ motif was required for partial recovery of growth of the $\Delta y b e Y$ mutant at $45^{\circ} \mathrm{C}$ and to rescue rRNA processing defects of the $\Delta y b e Y$ mutant, supporting the importance of this motif for YbeY function (Fig. 6B and C). Interestingly, changing the second and third His residues from this motif $(\mathrm{H} 118, \mathrm{H} 124)$ to Ala did not have much of an effect on complementation. A highly conserved Arg residue (R59) was also found to be necessary for growth complementation. The loss of complementation by H114A and R59A alleles was not due to insufficient protein expression as plasmid-driven expression of all $y b e Y$ mutant alleles exceeded $y b e Y$ expression from the endogenous locus (Fig. S3G). It should be noted that some of the ybeY mutant alleles that did not show an effect on complementation when expressed from a multicopy plasmid, may do so if they are expressed at levels of YbeY normally present in wild-type cells.

\section{Discussion}

We have shown that the functionally conserved UPF0054 protein family member, YbeY, influences rRNA maturation and ribosome formation in $E$. coli. Deletion of $y b e Y$ from $E$. coli results in defects in ribosome assembly and activity as well as in attenuation of 16S, $23 \mathrm{~S}$ and 5S rRNA processing.

rRNA processing, ribosome maturation and translation are intertwined. Several reports have implicated partial ribosome assembly as a requirement for full rRNA 


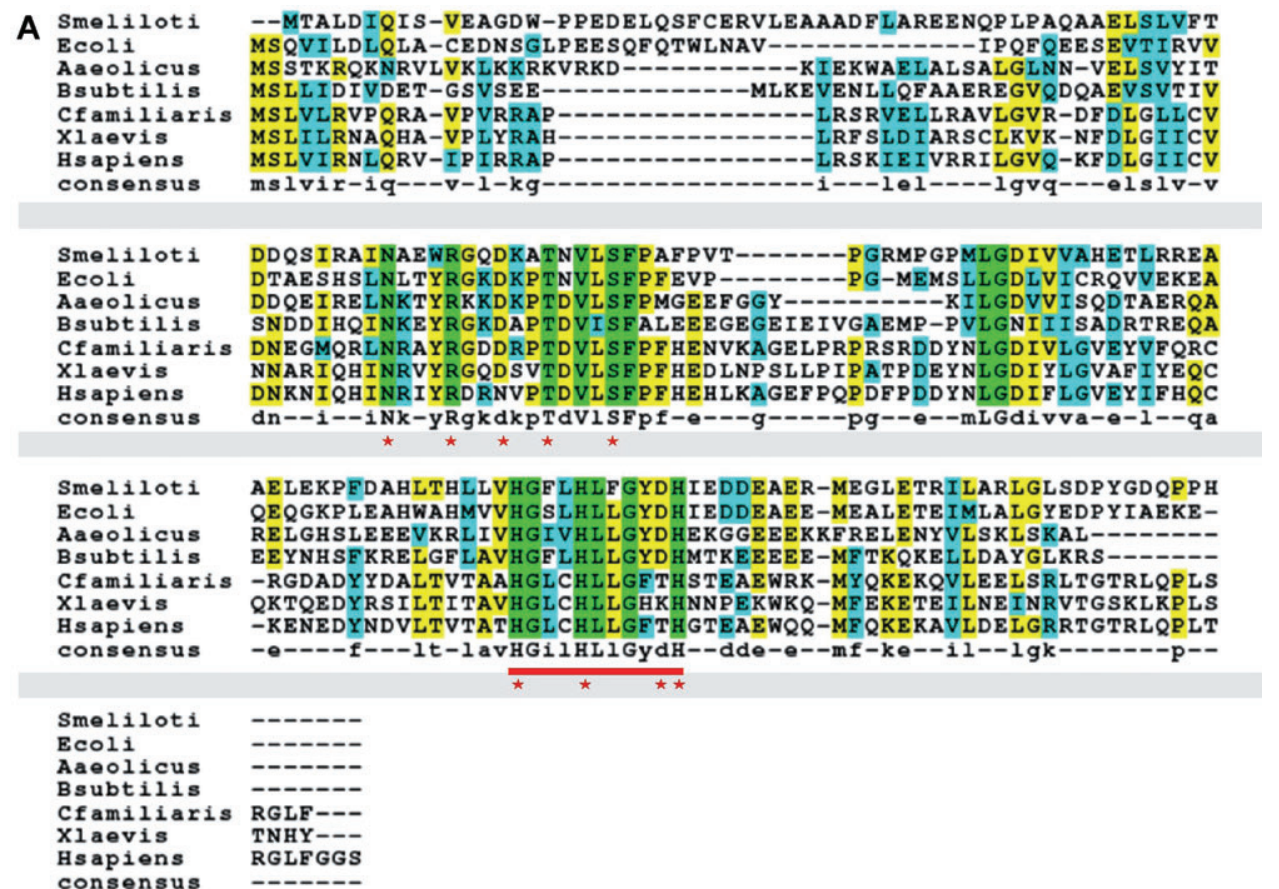

B

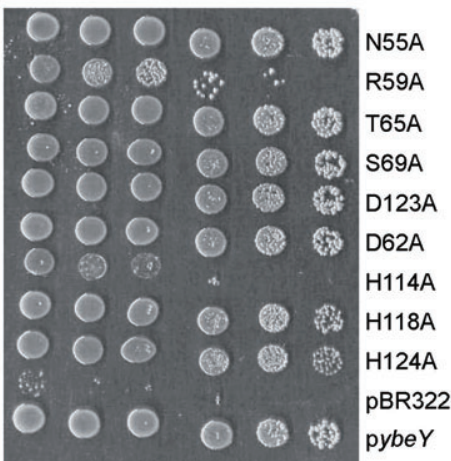

C

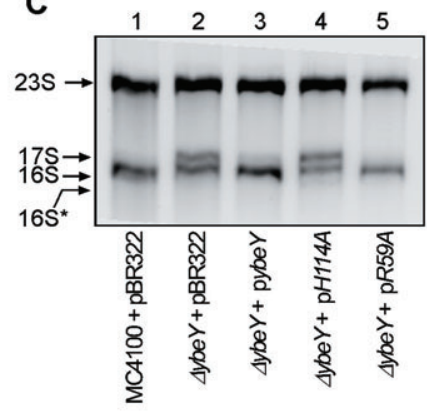

Fig. 6. A. Sequence alignment of UPF0054 homologues from bacteria and eukaryotes. Alignments were performed using T-coffee (Poirot et al., 2003). The red bar underlines the conserved H3XH5XH motif that is used to classify members of this family. Red asterisks indicate amino acid residues that were analysed in this study by Ala mutagenesis.

B. Sensitivity of the $\Delta y b e Y$ mutant strain expressing mutant $y b e Y$ alleles to high temperature. Strains were serially diluted (1:10), plated on LB plates and incubated at $45^{\circ} \mathrm{C}$.

C. rRNA profiles of the $\triangle y b e Y$ mutant expressing the mutant $y b e Y$ alleles H114A and R59A. The positions of 23S, 17S, 16S and 16S* rRNAs are indicated.

maturation (Mangiarotti et al., 1974; 1975; Srivastava and Schlessinger, 1988) and, while deletion of specific RNases can lead to specific defects in rRNA maturation (Li and Deutscher, 1995; Li et al., 1999a,b), loss of nonRNase ribosome maturation factors can also lead to rRNA maturation defects. For example, both Era and ObgE are GTPases required for ribosome assembly. Era and ObgE are essential for viability in E. coli and loss of either activity causes accumulation of 17S rRNA (Inoue et al., 2003; Sato et al., 2005). Similarly, deletion of ribosome maturation factors RimB and RimP results in slower growth rates, reduced number of polysomes and accumu- lation of 16S rRNA precursor (Lövgren et al., 2004). Furthermore, loss of RsgA, a GTPase activated by the $30 \mathrm{~S}$ ribosomal subunit, results in the accumulation of both $17 \mathrm{~S}$ rRNA and another species shorter than 16S rRNA (Himeno et al., 2004). Our results could also mean that loss of YbeY affects a critical step in ribosome biogenesis and thereby slows rRNA maturation. It will be interesting to explore physical interactions of $\mathrm{YbeY}$ with the ribosome and rRNA directly as well as possible effects of YbeY on ribosomal proteins, e.g. protein modifications. Supporting a potential association of $\mathrm{YbeY}$ with the ribosome, recent work has identified interactions between $\mathrm{YbeY}$ and both 
$30 \mathrm{~S}$ and $50 \mathrm{~S}$ ribosomal proteins in a large-scale pulldown experiment (Arifuzzaman et al., 2006).

Active translation has also been suggested to be required for certain steps in rRNA maturation (Hayes and Vasseur, 1976; Sirdeshmukh and Schlessinger, 1985; Srivastava and Schlessinger, 1988). The dramatic decrease in IF2 binding to the $30 \mathrm{~S}$ ribosomal subunit in the $\Delta y b e Y$ mutant (Fig. 2H) could suggest that $\mathrm{YbeY}$ is needed, directly or indirectly, to form the translation initiation complex. A decrease in efficiency of initiation complex formation could slow translation to a point that negatively impacts maturation of rRNA.

The strongest evidence of a role for YbeY in rRNA and/or ribosome maturation comes from the analysis of rRNA in strains carrying the $\triangle y b e Y$ mutation alone or in combination with other mutations in known RNases (Figs 3 and 4). RNases required for the processing of the $16 \mathrm{~S}$ rRNA 3 '-terminus, 23S rRNA 5'-terminus and 5S rRNA $5^{\prime}$-terminus have not been identified. Interestingly, we observe significant defects in processing of all of these termini in the $\triangle y b e Y$ mutant (Fig. $3 C$ and D). The $\Delta y b e Y$ mutant shows a much stronger overall effect on rRNA maturation than any of the RNases tested, even those with a known role in rRNA processing (Fig. $4 \mathrm{C}-\mathrm{H}$ ). Only RNase III, which is required for the initial cleavages of the rRNA precursor, also affects the maturation of multiple rRNA termini. Deletion of both $y b e Y$ and $r n c$ stabilizes the accumulation of an 18S-like rRNA precursor with similar mobility to that of a transient $18 \mathrm{~S}$ rRNA species previously observed in an rnc mutant (Gegenheimer et al., 1977). Loss of YbeY activity may therefore exacerbate a specific rRNA maturation defect that is only observed in the absence of the RNase III required for the initial cleavage events in rRNA maturation.

Deletion of $y b e Y$ along with either rnr or pnp prevents, almost completely, the maturation of the 16S rRNA 3 '-terminus (Fig. 4D), while maturation of all other rRNA termini proceeds to some degree in both of these mutants. Furthermore, large amounts of $16 \mathrm{~S}$ rRNA are improperly processed in the $\Delta y b e Y \Delta r n r$ double mutant, as shown by the accumulation of additional aberrant $16 \mathrm{~S}$ rRNA maturation intermediates. These results suggest that $\mathrm{YbeY}$ may function along with RNase $\mathrm{R}$ and PNPase in the maturation of the 16S rRNA $3^{\prime}$-terminus and potentially in other steps of rRNA metabolism such as rRNA quality control.

Site-directed mutagenesis of YbeY supports the requirement for one of the His residues (H114) in the highly conserved putative hydrolase domain and a highly conserved Arg residue (R59) for YbeY function in vivo (Fig. 6). For example, ectopic expression from a plasmid of $y b e Y$ mutants H114A and R59A in the $\triangle y b e Y$ mutant failed to complement the growth defect of the mutant strain at elevated temperatures. The $\mathrm{H} 3 \mathrm{XH} 5 \mathrm{XH}$ motif is postulated to co-ordinate a $\mathrm{Zn}^{2+}$ ion that could act catalytically in a hydrolysis reaction (Zhan et al., 2005). The crystal structure of YbeY also shows the highly conserved R59 pointing inward towards the H3XH5XH motif (Zhan et al., 2005). The location of R59 along with its positive charge could imply that R59 helps position a substrate near the $\mathrm{H} 3 \mathrm{XH} 5 \mathrm{XH}$ motif. Given the strong genetic interactions of $y b e Y$ with $r n c, r n r$ and pnp (Fig. 4C-H) established here, along with the requirement of the putative hydrolase domain for $y b e Y$ activity, YbeY may also have RNase activity in the cell.

It is currently unclear why only one (H114) out of three His residues within the highly conserved $\mathrm{H} 3 \mathrm{XH} 5 \mathrm{XH}$ motif appears to be required for $y b e Y$ activity. It is possible that contributions of the other two His residues (H118, H124) towards YbeY activity may also be seen, when YbeY mutants are expressed at wild type-like levels. It is interesting to note the H114A and R59A mutants show similar sensitivity to growth at elevated temperature, but the rRNA processing defects are markedly different (Fig. 6B and $C$ ). While the rRNA profile of the H114A mutant resembles that of the $y b e Y$ mutant, the rRNA profile of the R59A mutant more closely resembles that of the MC4100, suggesting that the different mutations affect different functions of YbeY or affect the same rRNA processing function to different degrees.

Finally, although deletion of ybeY results in severe rRNA maturation defects suggesting a role in rRNA and/or ribosome maturation it remains possible that $\mathrm{YbeY}$ actually has an alternative function in the cell that impacts these processes. Although this remains a formal possibility, the simplest explanation for the striking effects of deletion of $y b e Y$ on rRNA processing and ribosome assembly is that YbeY functions directly in rRNA and/or ribosome maturation and assembly.

\section{Experimental procedures}

\section{Strains, plasmids, growth conditions and DNA manipulations}

Strains and plasmids are shown in Table S1. Strains were grown aerobically in Luria-Bertani (LB) medium at $37^{\circ} \mathrm{C}$ except for heat shock experiments where strains were grown at $45^{\circ} \mathrm{C}$. Antibiotics for strain selection were used at the following concentrations, ampicillin $100 \mu \mathrm{g} \mathrm{ml}^{-1}$, chloramphenicol $20 \mu \mathrm{g} \mathrm{ml}^{-1}$ and kanamycin $25 \mu \mathrm{g} \mathrm{ml}^{-1}$. Deletion of $y b e Y$ and the addition of a C-terminal FLAG tag to the genomic copy of $y b e Y$ (strain BWD55) was performed using the methods of Wanner (Datsenko and Wanner, 2000). For deletion, $y b e Y$ was first replaced with a cat ${ }^{\mathrm{R}}$ cassette creating the $\Delta y b e Y:: c^{\mathrm{R}}{ }^{\mathrm{R}}$ mutant. The cat $^{\mathrm{R}}$ cassette was then excised to create a clean $\triangle y b e Y$ deletion mutant in MC4100. Deletion of $y b e Y$ maintains the ATG start of $y b e Y$ and the overlapping stop codon of the upstream gene $y b e Z$. Translation from the ATG start of $y b e Y$ terminates eight amino acids into the flip 
recombinase site of the $\lambda$-red gene targeting recombinase system (Datsenko and Wanner, 2000). DNA primers used for deletion of $y b e Y$ and addition of the C-terminal FLAG tag are listed in Table S2. Allele transfers were done by $\mathrm{P} 1$ transduction. DNA manipulations were performed according to the methods of Sambrook (Sambrook and Russell, 2001). Site-directed mutagenesis was performed using the Quikchange II kit from Stratagene. DNA primers used for site-directed mutagenesis are listed in Table S2. For complementation studies, ybeY, SMc01113 and yqfG were cloned downstream of the tetracycline promoter in pBR322 or the tryptophan promoter in pMS03. The DNA primers used for complementation cloning are shown in Table S2.

\section{Phenotypic analysis}

Stress and plant assays were performed as previously described (Davies and Walker, 2008).

\section{Polysome and rRNA analysis}

rRNA and polysomes were extracted from cells growing exponentially at $37^{\circ} \mathrm{C}$, unless otherwise stated. Cells were pelleted, resuspended in buffer A (20 mM HEPES pH 7.5, $5 \mathrm{mM} \beta$-mercaptoethanol, $10 \mathrm{mM} \mathrm{MgCl}_{2}, 50 \mathrm{mM} \mathrm{NH}_{4} \mathrm{Cl}$ and $0.1 \mathrm{mM}$ PMSF) and lysed by repeated freeze/thaw cycles. After clarification by centrifugation, lysates were loaded on a $5-20 \%$ sucrose gradient. Polysomes and ribosomes were separated by ultracentrifugation followed by fractionation using a peristaltic pump. The $A_{260}$ value was determined for each fraction.

rRNA was extracted from logarithmically growing cultures or separated ribosome fractions using Qiagen RNeasy Mini Kit. For gross analysis of $16 \mathrm{~S}$ and $23 \mathrm{~S}$ rRNA, $800 \mathrm{ng}$ of rRNA was separated by synergel/agarose gel electrophoresis as described (Wachi et al., 1999) using $0.9 \%$ synergel and $0.7 \%$ agarose.

Primer extension assays were performed using Superscript II reverse transcriptase (Invitrogen) as per manufacturer's instructions with primers annealing to the $5^{\prime}$-terminal region of mature $16 \mathrm{~S}, 23 \mathrm{~S}$ and $5 \mathrm{~S}$ rRNA respectively. The site-specific RNase $\mathrm{H}$ cleavage assay was performed as described (Li and Deutscher, 1995; Li et al., 1999a,b) followed by Northern hybridization using probes specific for the mature 3'-termini of 16S, 23S and 5S rRNAs respectively. One hundred nanograms of total RNA was used for each assay. Reaction products were separated on denaturing polyacrylamide gels; $10 \%$ polyacrylamide/7 $\mathrm{M}$ urea for primer extensions and $6 \%$ polyacrylamide/7 $\mathrm{M}$ urea for site-specific $\mathrm{RNase} \mathrm{H}$ cleavage assays. DNA oligonucleotides for primer extension and RNase $\mathrm{H}$ mapping are shown in Table S2.

For the Northern blot in Fig. 2B, 250 ng of total MC4100 and $\triangle y b e Y$ RNA was separated on a $1 \%$ agarose gel containing $2 \%$ formamide. Probes used to identify $17 \mathrm{~S}$ rRNA $5^{\prime}$ and $3^{\prime}$-termini are shown in Table S2. The probes were detected with the AlkPhos Direct Labeling Kit (Amersham).

For temperature shift assays, cultures were grown to exponential phase at $37^{\circ} \mathrm{C}$. Samples were taken for rRNA analysis and the cultures were then shifted to $45^{\circ} \mathrm{C}$. After $1 \mathrm{~h}$ samples were taken for rRNA analysis.
In vitro translation and lacZ assays

In vitro translation assays were performed essentially as described (Matthaei and Nirenberg, 1961). Exponentially growing cells were pelleted, resuspended in buffer $A$ and lysed by French press. Lysates were centrifuged twice at $30000 \mathrm{~g}$ and once at $100000 \mathrm{~g}$. The resulting supernatant was kept as the $\mathrm{S} 100$ fraction, and the pelleted ribosomes were washed and resuspended in buffer A. Ribosomes were further purified by pelleting at $200000 \mathrm{~g}$ through a $20 \%$ sucrose cushion. Pelleted ribosomes were resuspended in buffer A and quantified by UV absorbance at $260 \mathrm{~nm}$, and stored at $-80^{\circ} \mathrm{C}$.

Mix I ( $25 \mu \mathrm{g}$ of polyU mRNA and 10 pmoles of ribosomes) was pre-incubated at $37^{\circ} \mathrm{C}$ for $15 \mathrm{~min}$ in polyU-mix $(20 \mathrm{mM}$ HEPES pH 7.5, $2 \mathrm{mM}$ DTT, $10 \mathrm{mM} \mathrm{MgCl}_{2}, 50 \mathrm{mM} \mathrm{NH}_{4} \mathrm{Cl}$, $1 \mathrm{mM}$ spermidine, $2 \mathrm{mM}$ ATP, $0.5 \mathrm{mM}$ GTP, $8 \mathrm{mM}$ phosphoenolpyruvate and $2 \mu \mathrm{M}$ pyruvate kinase). Mix II (50 $\mu \mathrm{g}$ of

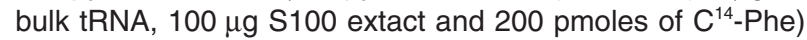
was incubated at $37^{\circ} \mathrm{C}$ for $15 \mathrm{~min}$ in polyU-mix. Mix I and II were mixed and incubated at $37^{\circ} \mathrm{C}$ for $15 \mathrm{~min} .{ }^{14} \mathrm{C}$-Phe incorporation into protein was determined by TCA precipitation and scintillation counting. All reactions were performed in triplicate.

For LacZ assays, strains carrying wild-type or mutant lacZ alleles on a plasmid were harvested in exponential phase. The assay for LacZ activity was then performed as described (Miller, 1972). The per cent LacZ activity (in Miller units) of each mutant lac $Z$ allele relative to the wild-type lac $Z$ allele for MC4100 or the $\Delta y b e Y$ mutant is reported.

\section{Acknowledgements}

We thank Dr Harry Noller for helpful discussion, Dr Murray Deutscher for his generous gifts of $r n b, r n d, r p h, r n t, r n e-1$ and $r n r$ E. coli mutant strains and Dr Albert Dahlberg for insightful discussion and for his gift of plasmids pSG25, pSG163, pSG853, pSG3/4, plac7 and plac10. This work was supported by National Institutes of Health Grant GM31030 to G.C.W. and Grant GM17151 to U.L.R., MIT Center for Environmental Health Sciences Grant P30ES002109, a National Sciences and Engineering Research Council of Canada graduate scholarship to B.W.D. and an $\mathrm{NCl}$ postdoctoral fellowship to L.A.S. G.C.W. is an American Cancer Society Research Professor.

\section{References}

Akerley, B.J., Rubin, E.J., Novick, V.L., Amaya, K., Judson, N., and Mekalanos, J.J. (2002) A genome-scale analysis for identification of genes required for growth or survival of Haemophilus influenzae. Proc Natl Acad Sci USA 99: 966971.

Arifuzzaman, M., Maeda, M., Itoh, A., Nishikata, K., Takita, C., Saito, R., et al. (2006) Large-scale identification of protein-protein interaction of Escherichia coli K-12. Genome Res 16: 686-691.

Bunner, A.E., Nord, S., Wikström, P.M., and Williamson, J.R. (2010) The effect of ribosome assembly cofactors on in vitro $30 \mathrm{~S}$ subunit reconstitution. $\mathrm{J} \mathrm{Mol} \mathrm{Biol} 398$ : 1-7. 
Cheng, Z.F., and Deutscher, M.P. (2003) Quality control of ribosomal RNA mediated by polynucleotide phosphorylase and RNase R. Proc Natl Acad Sci USA 100: 6388-6393.

Datsenko, K.A., and Wanner, B.L. (2000) One-step inactivation of chromosomal genes in Escherichia coli K-12 using PCR products. Proc Natl Acad Sci USA 97: 6640-6645.

Davies, B.W., and Walker, G.C. (2008) A highly conserved protein of unknown function is required by Sinorhizobium meliloti for symbiosis and environmental stress protection. $J$ Bacteriol 190: 1118-1123.

Deutscher, M.P. (2009) Maturation and degradation of ribosomal RNA in bacteria. Prog Mol Biol Transl Sci 85: 369391.

Friedberg, E.C., Walker, G.C., Siede, W., Wood, R.D., Schultz, R.A., and Ellenberger, T. (2005) DNA Repair and Mutagenesis. Washington, DC: ASM Press.

Gegenheimer, P., Watson, N., and Apirion, D. (1977) Multiple pathways for primary processing of ribosomal RNA in Escherichia coli. J Biol Chem 252: 3064-3073.

Gil, R., Silva, F.J., Pereto, J., and Moya, A. (2004) Determination of the core of a minimal bacterial gene set. Microbiol Mol Biol Rev 68: 518-537.

Green, R., and Noller, H.F. (1999) Reconstitution of functional $50 S$ ribosomes from in vitro transcripts of Bacillus stearothermophilus 23S rRNA. Biochemistry 38: 1772-1779.

Hayes, F., and Vasseur, M. (1976) Processing of the 17-S Escherichia coli precursor RNA in the 27-S pre-ribosomal particle. Eur J Biochem 61: 433-442.

Himeno, H., Hanawa-Suetsugu, K., Kimura, T., Takagi, K., Sugiyama, W., Shirata, S., et al. (2004) A novel GTPase activated by the small subunit of ribosome. Nucl Acids Res 32: 5303-5309.

Imlay, J.A., and Linn, S. (1987) Mutagenesis and stress responses induced in Escherichia coli by hydrogen peroxide. J Bacteriol 169: 2967-2976.

Inoue, K., Alsina, J., Chen, J., and Inouye, M. (2003) Suppression of defective ribosome assembly in a rbfA deletion mutant by overexpression of Era, an essential GTPase in Escherichia coli. Mol Microbiol 48: 1005-1016.

Jones, K.M., Kobayashi, H., Davies, B.W., Taga, M.E., and Walker, G.C. (2007) How rhizobial symbionts invade plants: the Sinorhizobium-Medicago model. Nat Rev Microbiol 5: 619-633.

Kaczanowska, M., and Ryden-Aulin, M. (2007) Ribosome biogenesis and the translation process in Escherichia coli. Microbiol Mol Biol Rev 71: 477-494.

King, T.C., Sirdeshmukh, R., and Schlessinger, D. (1984) RNase III cleavage is obligate for maturation but not for function of Escherichia coli pre-23S rRNA. Proc Natl Acad Sci USA 81: 185-188.

Kobayashi, K., Ehrlich, S.D., Albertini, A., Amati, G., Andersen, K.K., Arnaud, M., et al. (2003) Essential Bacillus subtilis genes. Proc Natl Acad Sci USA 100: 4678-4683.

$\mathrm{Li}$, Z., and Deutscher, M.P. (1995) The tRNA processing enzyme RNase $T$ is essential for maturation of 5S RNA. Proc Natl Acad Sci USA 92: 6883-6886.

Li, Z., Pandit, S., and Deutscher, M.P. (1999a) RNase G (CafA protein) and RNase $E$ are both required for the $5^{\prime}$ maturation of $16 \mathrm{~S}$ ribosomal RNA. EMBO J 18: 28782885.

Li, Z., Pandit, S., and Deutscher, M.P. (1999b) Maturation of
23S ribosomal RNA requires the exoribonuclease RNase T. RNA 5: 139-146.

Lövgren, J.M., Bylund, G.O., Srivastava, M.K., Lundberg, L.A., Persson, O.P., Wingsle, G., and Wikström, P.M. (2004) The PRC-barrel domain of the ribosome maturation protein RimM mediates binding to ribosomal protein S19 in the 30 S ribosomal subunits. RNA 10: 1798-1812.

Mangiarotti, G., Turco, E., Ponzetto, A., and Altruda, F. (1974) Precursor $16 \mathrm{~S}$ RNA in active $30 \mathrm{~S}$ ribosomes. Nature 247: 147-148.

Mangiarotti, G., Turco, E., Perlo, C., and Altruda, F. (1975) Role of precursor 16S RNA in assembly of E. coli $30 \mathrm{~S}$ ribosomes. Nature 253: 569-571.

Matthaei, H., and Nirenberg, M.W. (1961) The dependence of cell-free protein synthesis in E. coli upon RNA prepared from ribosomes. Biochem Biophys Res Commun 4: 404408.

Miller, J.H. (1972) Experiments in Molecular Cloning. Cold Spring Harbor, NY: Cold Spring Harbor Laboratory.

Misra, T.K., and Apirion, D. (1979) RNase E, an RNA processing enzyme from Escherichia coli. J Biol Chem 254: 11154-11159.

Nierhaus, K.H. (1980) The assembly of the prokaryotic ribosome. Biosystems 12: 273-282.

Nierhaus, K.H. (1991) The assembly of prokaryotic ribosomes. Biochimie 73: 739-755.

Noller, H.F., and Nomura, M. (1987) Ribosomes. Eschericha coli and Salmonella typhimurium; Cellular and Molecular Biology. Washington DC: American Society for Microbiology, pp. 104-125.

Nomura, M., and Erdmann, V.A. (1970) Reconstitution of $50 S$ ribosomal subunits from dissociated molecular components. Nature 228: 744-748.

Oganesyan, V., Busso, D., Brandsen, J., Chen, S., Jancarik, J., Kim, R., and Kim, S.H. (2003) Structure of the hypothetical protein AQ_1354 from Aquifex aeolicus. Acta Crystallogr D Biol Crystallogr 59: 1219-1223.

Poirot, O., O'Toole, E., and Notredame, C. (2003) Tcoffee@ igs: a web server for computing, evaluating and combining multiple sequence alignments. Nucl Acids Res 31: 35033506.

Rasouly, A., Schonbrun, M., Shenhar, Y., and Ron, E.Z. (2009) YbeY - a heat shock protein involved in translation in Escherichia coli. J Bacteriol 191: 2649-2655.

Robertson, H.D., Webster, R.E., and Zinder, N.D. (1967) A nuclease specific for double-stranded RNA. Virology 32: 718-719.

Sambrook, J., and Russell, D.W. (2001) Molecular Cloning. A Laboratory Manual. Cold Spring Harbour, NY: Cold Spring Harbor Laboratory.

Sato, A., Kobayashi, G., Hayashi, H., Yoshida, H., Wada, A., Maeda, M., et al. (2005) The GTP binding protein Obg homolog ObgE is involved in ribosome maturation. Genes Cells 10: 393-408.

Sirdeshmukh, R., and Schlessinger, D. (1985) Why is processing of $23 \mathrm{~S}$ ribosomal RNA in Escherichia coli not obligate for its function? J Mol Biol 186: 669-672.

Srivastava, A.K., and Schlessinger, D. (1988) Coregulation of processing and translation: mature $5^{\prime}$ termini of Escherichia coli $23 \mathrm{~S}$ ribosomal RNA form in polysomes. Proc Natl Acad Sci USA 85: 7144-7148. 
Tatusov, R.L., Natale, D.A., Garkavtsev, I.V., Tatusova, T.A., Shankavaram, U.T., Rao, B.S., et al. (2001) The COG database: new developments in phylogenetic classification of proteins from complete genomes. Nucl Acids Res 29: 22-28.

Verma, R., Aravind, L., Oania, R., McDonald, W.H., Yates, J.R., 3rd, Koonin, E.V., and Deshaies, R.J. (2002) Role of Rpn11 metalloprotease in deubiquitination and degradation by the 26S proteasome. Science 298: 611-615.

Wachi, M., Umitsuki, G., Shimizu, M., Takada, A., and Nagai, K. (1999) Escherichia coli cafA gene encodes a novel RNase, designated as RNase $G$, involved in processing of the $5^{\prime}$ end of $16 \mathrm{~S}$ rRNA. Biochem Biophys Res Commun 259: 483-488.

Wilson, D.N., and Nierhaus, K.H. (2007) The weird and wonderful world of bacterial ribosome regulation. Crit Rev Biochem Mol Biol 42: 187-219.

Wireman, J.W., and Sypherd, P.S. (1974) In vitro assembly of $30 \mathrm{~S}$ ribosomal particles from precursor 16S RNA of Escherichia coli. Nature 247: 552-554.
Yeh, D.C., Parsons, L.M., Parsons, J.F., Liu, F., Eisenstein, E., and Orban, J. (2005) NMR structure of HI0004, a putative essential gene product from Haemophilus influenzae, and comparison with the X-ray structure of an Aquifex aeolicus homolog. Protein Sci 14: 424-430.

Zhan, C., Fedorov, E.V., Shi, W., Ramagopal, U.A., Thirumuruhan, R., Manjasetty, B.A., et al. (2005) The ybeY protein from Escherichia coli is a metalloprotein. Acta Crystallograph Sect F Struct Biol Cryst Commun 61: 959-963.

\section{Supporting information}

Additional supporting information may be found in the online version of this article.

Please note: Wiley-Blackwell are not responsible for the content or functionality of any supporting materials supplied by the authors. Any queries (other than missing material) should be directed to the corresponding author for the article. 